\title{
Three-dimensional Characterization of Human Ventricular Myofiber Architecture by Ultrasonic Backscatter
}

\author{
Samuel A. Wickline, ${ }^{\star}$ Edward D. Verdonk, ${ }^{\star}$ and James G. Miller \\ Washington University ${ }^{*}$ School of Medicine and ${ }^{\ddagger}$ Department of Physics, St. Louis, Missouri 63110
}

\begin{abstract}
Normal human left ventricular architecture comprises a highly aligned array of cardiac myofibers whose orientation depends on transmural location. This study was designed to determine whether measurement of integrated backscatter could be used detect the progressive transmural shift of myofiber alignment that occurs from epicardium to endocardium in human ventricular wall segments. Integrated backscatter was measured at 32 transmural levels in seven cylindrical biopsy specimens $(1.4 \mathrm{~cm}$ diam) sampled from normal regions of six explanted fixed human hearts by insonification of samples at 180 independent angles in $2^{\circ}$ steps around their entire circumference with a 5MHz broadband piezoelectric transducer. Histologic analysis was performed to determine fiber orientation. Integrated backscatter varied approximately as a sinusoidal function of the angle of insonification at each transmural level. Greater integrated backscatter was observed for insonification perpendicular as compared with parallel to fibers (difference $=14.5 \pm 0.6$ dB). Ultrasonic analysis revealed a progressive transmural shift in fiber orientation of $\sim 9.2 \pm 0.7$ degrees $/ \mathrm{mm}$ of tissue. Histologic analysis revealed a concordant shift in fiber orientation of $7.9 \pm 0.8$ degrees $/ \mathrm{mm}$ of tissue. Thus, human myocardium manifests anisotropy of ultrasonic scattering that may be useful for characterization of the intramural fiber alignment and overall three-dimensional organization of cardiac myofibers. (J. Clin. Invest. 1991. 88:438-446.) Key words: ultrasonic tissue characterization - transmural cardiac structure • ultrasonic anisotropy • cardiac myofibers
\end{abstract}

\section{Introduction}

The architecture of the normal left ventricular wall comprises a highly aligned continuum of myofibers whose orientation relative to the major axis of the ventricle depends on their transmural location. Streeter et al. have demonstrated that the predominant orientation of fibers shifts through an angle of $\sim 120^{\circ}$ from epicardium to endocardium $(1,2)$. Midmyocardial fibers maintain a predominantly circumferential orientation, whereas epicardial fibers course obliquely toward the apex

This work was presented in part as an abstract at the American Heart Association Meeting, 12 November 1990, Dallas, TX.

Address reprint requests to Samuel A. Wickline, M.D., Cardiovascular Division, Washington University School of Medicine, Box 8086, 660 South Euclid Ave., St. Louis, MO 63110.

Received for publication 2 October 1990 and in revised form 24 January 1991.

J. Clin. Invest.

(c) The American Society for Clinical Investigation, Inc.

0021-9738/91/08/0438/09 \$2.00

Volume 88, August 1991, 438-446 and endocardial fibers course obliquely toward the base. These observations have led to a more thorough understanding of the mechanical function of the heart and have facilitated the development of realistic mathematical models of ventricular systolic and diastolic function that incorporate physiologic material elastic and geometric properties (3-6). Pathologic alterations of this complex myocardial architecture caused by infarction or cardiomyopathy often culminate in congestive heart failure and ventricular arrhythmias. Unfortunately, available clinical diagnostic methods do not permit sensitive noninvasive characterization of myocardial intramural architecture.

Our laboratory and others have reported previously that quantitative ultrasonic characterization of myocardial tissue can provide important information about the physical composition of the left ventricle, independent of its wall motion or chamber dimension (7-15). We have shown that quantitative indexes of ultrasonic integrated backscatter and attenuation derived from analysis of radio frequency $(\mathrm{rf})^{1}$ data can differentiate normal myocardial tissue from cardiomyopathic, infarcted, and stunned tissue in experimental animals and in patients (16-19). Recently, we have demonstrated that the magnitudes of backscatter and attenuation depend on the angle of insonification of the left ventricular wall with respect to the predominant orientation of myofibers in canine heart $(20,21)$. This directional dependence of ultrasonic properties is referred to as anisotropy.

The clinical relevance of ultrasonic anisotropy is apparent to any practiced echocardiographer who has observed the phenomenon of lateral and septal wall echo "drop out" in parasternal short-axis two-dimensional echocardiographic images. Aygen and Popp have reported that clinical echocardiographic images of human heart tissue manifest qualitative features of anisotropy (22). However, quantitative characterization of the ultrasonic anisotropic properties of human myocardium has not been reported to our knowledge.

To determine whether ultrasonic interrogation of human myocardium manifests a quantitative dependence on myofiber orientation, we studied the anisotropic properties of biopsy specimens of explanted fixed human hearts. We hypothesized that measurement of backscatter would permit detection of the progressive transmural shift in the alignment of myofibers from epicardium to endocardium. Acquisition of these data represents an important preliminary step toward the clinical application of ultrasonic tissue characterization for delineation of the three-dimensional organization of normal and pathologic cardiac structure.

\section{Methods}

Pathologic specimens. Seven pathologic specimens of fixed human heart tissue were obtained from explanted formalin-fixed native hearts

1. Abbreviation used in this paper: $\mathrm{rf}$, radio frequency. 
of six patients who underwent cardiac allograft transplantation for congestive ischemic cardiomyopathy at our institution within the past $5 \mathrm{yr}$. Regions of grossly normal tissue remote from areas of infarction were identified by visual inspection. A No. 10 cork borer was used to remove transmural cylindrical plugs of tissue $(1.4 \mathrm{~cm}$ diam) whose principal axes were orthogonal to the epicardial surface. The approximate orientation of each plug with respect to the ventricular apex was marked along its lateral aspect with ink after excision. The irregular endocardial trabeculations were shaved off with a razor blade. The plugs were mounted flush on the end of 2-cm-long styrofoam cylindrical templates of equivalent diameter by gluing the endocardial surface to the template with cyanoacrylate. The styrofoam templates served as convenient tissue holders of relatively low reflectivity that did not interfere with the ultrasonic interrogation of the tissue samples (see Results).

Acquisition of ultrasonic data. Tissue plugs were interrogated with ultrasound by immersion in the water bath of a computer-controlled scanning apparatus. All ultrasonic measurements were conducted at room temperature $\left(\sim 20^{\circ} \mathrm{C}\right)$. Insonification was accomplished with a $5-\mathrm{MHz}$ focused piezoelectric transducer (0.5-in diameter, 2-in focal length; model V309; Panametrics, Inc., Waltham, MA). The tissuestyrofoam assembly was suspended from a rotating stage and oriented so that the ultrasound beam was parallel to epicardial and endocardial surfaces. The focal zone of the transducer was positioned at the front surface of each cylindrical tissue specimen. Ultrasonic rf pulses were launched from the transducer with a Metrotek pulser (model 215; Stavely Instruments, Kennewick, WA) and traveled through the water before interacting with tissue. Backscattered $\mathrm{rf}$ was amplified with a Metrotek receiver (model 106) and a segment of the rf trace corresponding to the central portion of the tissue sample (14 $\mu$ s round-trip, or $\sim 10.8 \mathrm{~mm}$ of tissue) was gated out with a Metrotek stepless gate (model 701) and passed to a spectrum analyzer $(30-\mathrm{kHz}$ bandwidth, 1-MHz/cm, 10 dB/division; model 8557A; Hewlett-Packard Co., Palo Alto, CA). The power spectral density data for each gated segment of rf was stored off-line under control of a Macintosh II computer outfitted with an IEEE-488 general-purpose interface bus (GPIB-NB; National Instruments, Austin, TX). Each power spectrum comprised 481 frequency bins in the range of $0-10 \mathrm{MHz}$.

Tissue plugs were interrogated from multiple angles at multiple transmural levels as depicted in Fig. 1. At each selected transmural level, the plugs were rotated around their centers in $2^{\circ}$ angular increments while the transducer remained fixed. These circumferential scans were initiated at the fiducial point previously marked to orient the plug to the ventricular apex. Fig. 2 illustrates typical backscattered If A-lines insonified perpendicular and parallel to the predominant fiber orientation in one transmural section of human tissue. Visual inspection of the raw $\mathrm{rf}$ data revealed greater scattering in the perpendicular direction for all transmural sections.

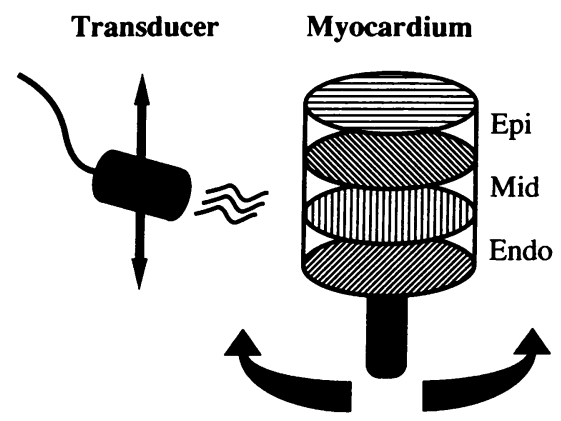

Figure 1. Experimental set-up for acquisition of rf data from cylindical tissue specimens. The tissue plug is rotated in $2^{\circ}$ increments to acquire if data from 180 independent angles of insonification. The transducer can move vertically with respect to the central axis of the tissue plug to interrogate selected transmural levels that manifest different fiber orientations and horizontally to provide replicate samples for spatial averaging at each angle of insonification.

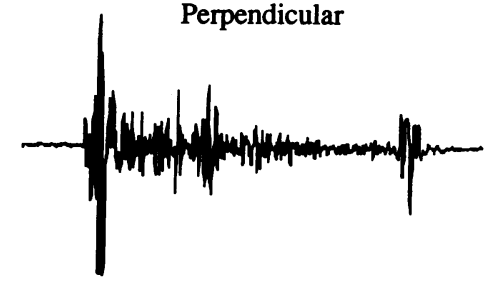

Figure 2. Backscattered rf data from human myocardial tissue. The top frame

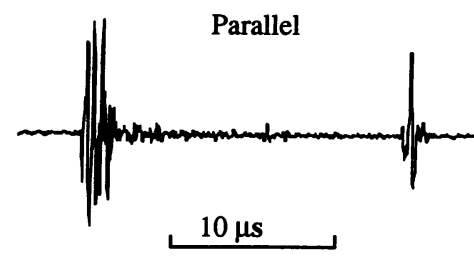
demonstrates typical raw if backscattered from tissue insonified perpendicular to the predominant fiber axis and the bottom frame shows backscatter from tissue insonified parallel to the fiber axis.

Power spectra were acquired sequentially from the gated if traces at each angle of insonification around the entire circumference of the cylindrical plug and integrated backscatter was computed for each power spectrum (see below). To provide spatial averaging at each angle of interrogation, five replications of rf power spectra were acquired adjacent to and including the central scan line at each angle by moving the transducer laterally in 1-mm steps first left then right. The values of integrated backscatter computed for the five power spectra at each angle of interrogation were averaged.

Circumferential scanning was initiated at least $1 \mathrm{~mm}$ below the epicardial surface and proceeded in increments of $1.5 \mathrm{~mm}$ toward the endocardial surface. Scans at each transmural level were initiated at the same fiducial point on the circumference of the plug to permit comparison of the angle dependence of backscatter at each level. After all transmural levels had been interrogated for a single plug, the plug was submitted for histologic analysis.

Analysis of ultrasonic data. The analysis of backscatter is based on a convolution model that equates convolutions of ultrasonic properties in the time domain with products in the frequency domain. The power spectrum of a signal scattered from a material that produces both scattering and attenuation can be written as $|E(f)|^{2}=\mid P(f)$ $\left.\right|^{2}|A(f)|^{2}|S(f)|^{2}$, where $|P(f)|^{2}$ is the power spectrum of an interrogating ultrasonic signal, $A(f)$ is a lumped parameter that represents attenuation suffered along the path to and within the gated volume (see Appendix I for further details), and $S(f)$ is the intrinsic backscatter transfer function of the material. A generalized substitution technique is used to produce a calibrated measurement of the scattering properties of the cardiac tissue $(23,24)$. By replacing the scattering material in the water tank with a reflector of known properties positioned at the focal point of the transducer, we can obtain a reference power spectrum $\left|E_{\text {red }}(f)\right|^{2}=|P(f)|^{2}\left|R_{\text {red }}(f)\right|^{2}$ determined only by the transducer power spectrum, $|P(f)|^{2}$, and the reflection coefficient of the planar reflector, $\boldsymbol{R}_{\text {red }}(f)$. In practice, the stainless steel plate used in this study is a nearly perfect reflector. Thus $\left|R_{\text {red }}(f)\right|^{2}$ will be taken as unity in the following formulation. By normalizing the spectrum measured from a scattering material to the spectrum from the "perfect" reflector and expressing the result on a logarithmic scale, we obtain the frequencydependent apparent backscatter transfer function $|B(f)|^{2}$ in units of decibels below the backscatter from a reflector from $10 \log |B(f)|^{2}=$ $10 \log \left(\left|E_{\text {tiesue }}(f)\right|^{2} /\left|E_{\text {ref }}(f)\right|^{2}\right)$. To generate a single relative index of backscatter efficiency, we calculate the frequency-averaged or integrated backscatter over the useful bandwidth of the transducer (typically, 3-7 MHz). This formulation assumes that transmission coefficients at specular boundaries are unity. Furthermore, backscatter measurements have not been compensated for tissue attenuation. Therefore, all ultrasonic measurements reported in this article represent "apparent" integrated backscatter. The measurement of apparent integrated backscatter is analogous to backscatter measurements that have been obtained in a clinical setting with the use of a real-time 
two-dimensional integrated backscatter imager developed by our laboratory $(16-19,25,26)$.

Integrated backscatter (spatially averaged) was computed for 180 independent angles in $2^{\circ}$ steps for each consecutive transmural level. Plots of integrated backscatter typically revealed a sinusoid-like dependence on the angle of insonification that comprised two peaks and two troughs for the amplitude of integrated backscatter per $360^{\circ}$ (see Results). The peak and trough values for integrated backscatter amplitude were determined from the plots and their averages were calculated.

A cross-correlation method was used to determine the shift of fiber orientation from one transmural section to the next. The correlation, $f(t)$, between two integrated backscatter functions from adjacent transmural levels, $L_{1}(\tau)$ and $L_{2}(\tau)$, is defined as

$f(t)=\int_{-\infty}^{\infty} \mathrm{L}_{1}(\tau+t) \mathrm{L}_{2}(\tau) \mathrm{d} \tau$,

where $L_{1}$ is displaced relative to $L_{2}$ by a lag $t$ (or angle, in our case). The maximum value for $f(t)$ represents the best fit between the two curves at some lag $t$ (or angular shift). The relative shift of angular position from consecutive scans was compensated for the thickness of tissue $(1.5 \mathrm{~mm})$ between each scan to yield an estimated angle of fiber shift per millimeter of tissue.

An intuitive appreciation for the cross-correlation operation may be gained by considering the correlation of a sine and a cosine function. If a sine wave is shifted through some angle $\alpha$ relative to a cosine wave which remains fixed in position, the sine wave will exactly overlap the cosine wave after a shift of $\pi / 2$ radians, or $90^{\circ}$. The correlation between the two waveforms $(f[t])$ will be maximum at an angular shift of $90^{\circ}$, and minimum at $-90^{\circ}$ where the sine and cosine functions are exactly $180^{\circ}$ out of phase. Thus, exact alignment of the two curves would require a shift of $90^{\circ}$ for one curve relative to the other because initially they are exactly $90^{\circ}$ out of phase. In our measurements, two similar (but not exact) sinusoidlike functions of scattering magnitude from different transmural levels are compared. We use the maximal value of the correlation to determine the angular shift that produces the greatest overlap between adjacent anisotropy functions. The calculated angular shift provides a measure of the rotation of a system of aligned myofibers from one intramural level to the next.

Histology. Each plug was removed from its styrofoam template and scored along one side with a scalpel at the fiducial mark that had been used to orient the plug with respect to the apex (or at the point where each of the circumferential ultrasound scans had been initiated). Each plug was cut into consecutive $1.5-2.5-\mathrm{mm}$-thick segments parallel to the epicardial surface so that the segments corresponded roughly to the transmural levels scanned by ultrasound. This method permitted accurate alignment of the ultrasound scans with the histologic sections. It was not always possible to slice segments thin enough to correspond exactly to each scan level, but enough sections were acquired from each plug to permit assessment of the shift in myofiber orientation from epicardium to endocardium. Each segment was embedded in paraffin and two sections ( $10 \mu \mathrm{m}$ thick) from each segment were stained with hematoxylin/eosin and with Masson trichrome stains.

All normal myocardial sections analyzed exhibited normal myofiber architecture by light microscopy. To provide a histologic control, a pathologic section was obtained from grossly infarcted tissue from one of the hearts. This section exhibited marked distortion of fiber architecture ranging from increased interstitial collagen to widespread deposition of scar tissue, and was easily differentiable from the normal sections.

The predominant orientation of myofibers in the normal sections stained with Masson trichrome were estimated by enlarging the tissue image and printing on $8 \times 10$-in photographic paper. The photographs were then scanned with a Hewlett-Packard ScanJet Plus flatbed scanner under control of a Macintosh II computer (Fig. 3). The scanned images then were analyzed with the use of image-processing software available from the National Institutes of Health (Image 1.29, Wayne Rasband, author). A pixel intensity thresholding function was used to

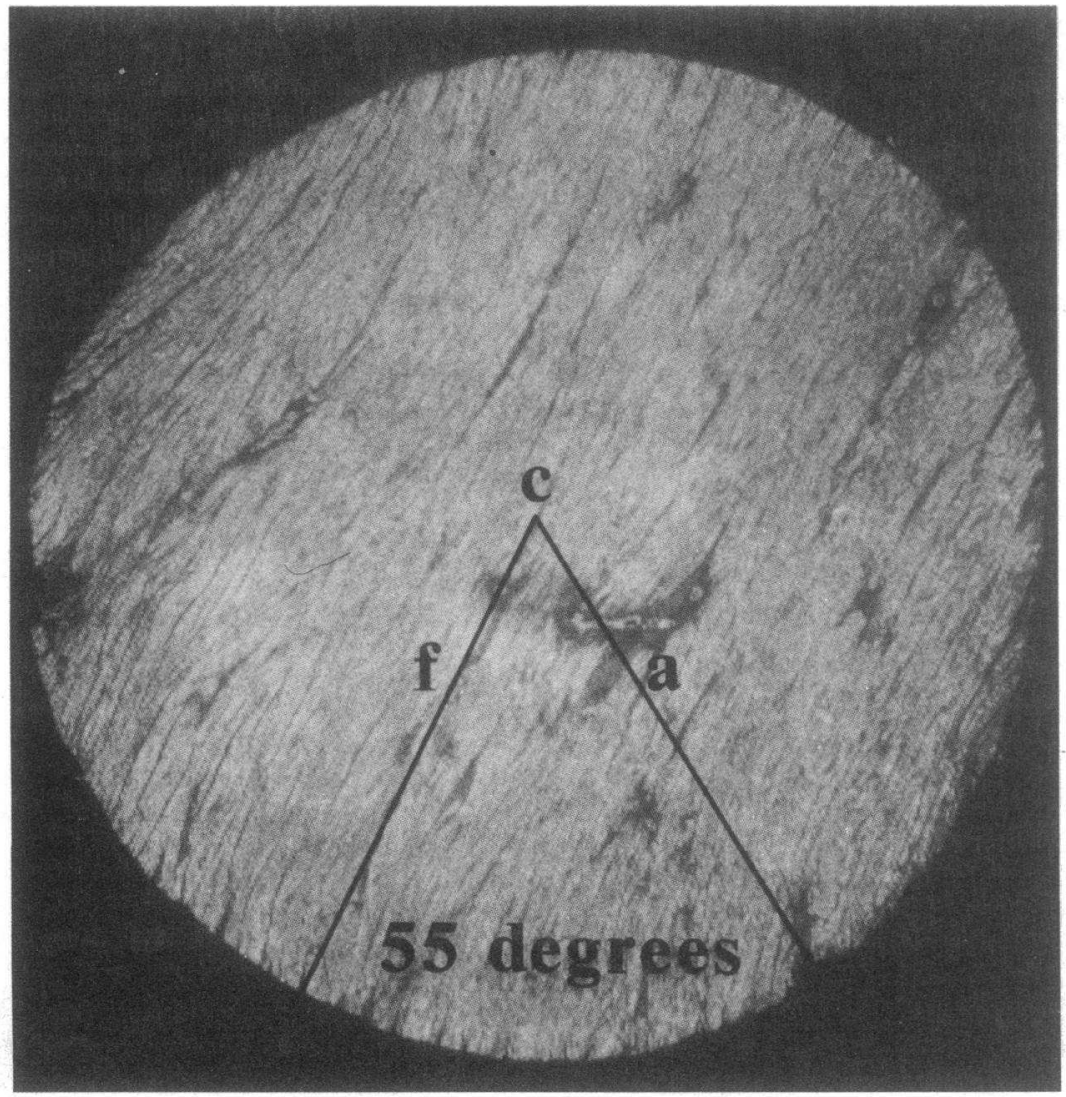

Figure 3. Histologic section at one transmural level from a cylindrical plug of myocardial tissue. The section is cut parallel to the epicardial surface of the plug and is $\sim 1.4 \mathrm{~cm}$ in diameter. The two rays $f-c$ and $c-a$ (along the fiber axis, $f$, through the center of mass of the tissue, $c$, to the apical reference mark, $a$ ) form an angle that can be used to compare ultrasonic and histologic measurements of fiber orientation. 
identify the boundaries of the tissue section, which permitted calculation of the center of mass. A line was drawn from the center of mass to the fiducial mark on the edge of the tissue. Another line was drawn from the center of mass along the predominant fiber axis, which was readily apparent in all tissue samples by gross inspection and by light microscopy. The angle between these two lines established the orientation of fibers with respect to the apical fiducial mark (Fig. 3). For each sequential section, the angle of orientation was computed and the overall difference in angular orientation between sections was recorded. The change in the angle of orientation between sections was compensated for the thickness of each section to yield the average shift in fiber angle per millimeter of tissue.

Statistical analysis. The measurements of transmural shifts of fiber orientation (degrees/mm of tissue) were compared for histologic and ultrasonic methods of analysis. Nonpaired $t$ tests (two-tailed) were used to test the significance of differences and statistical significance was attributed at the level of $P<0.05$. Standard error values are reported.

\section{Results}

Ultrasonic interrogation of human myocardium. A total of 32 transmural sections were studied. Three sections were excluded from analysis, two because of the presence of large epicardial coronary artery segments within the plane of ultrasonic interrogation that introduced artifact into the measurement of anisotropy and one endocardial section because it manifested a multiplicity of fiber orientations on histologic analysis. Fig. 2 illustrates typical backscattered rf A-lines for insonification perpendicular and parallel to the predominant fiber orientation in one transmural section. Visual inspection of the raw if data revealed greater magnitude of scattering for insonification perpendicular as compared with parallel to fibers for each transmural section. Fig. 4 illustrates the dependence of integrated backscatter on the angle of insonification for three transmural levels (epicardium, midmyocardium, and endocardium) in one tissue sample. The magnitude of backscatter manifested a sinusoidlike dependence on angle of insonification with a period of $\sim 180^{\circ}$. The average peak-to-nadir difference for integrated backscatter for all transmural sections from all human biopsy specimens was $14.5 \pm 0.6 \mathrm{~dB}$.

The positions of the peaks and nadirs of integrated backscatter also shifted progressively from epicardial to endocardial

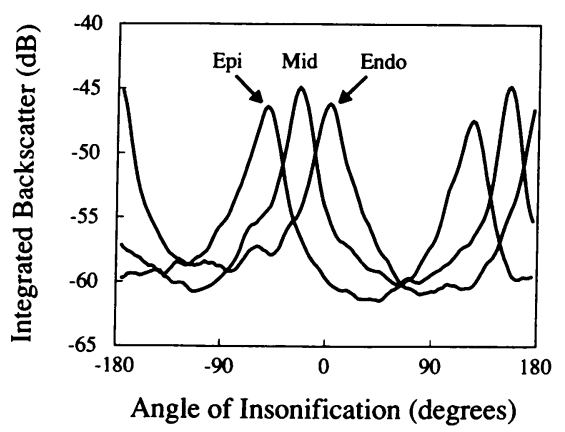

Figure 4. Apparent integrated backscatter (not compensated for attenuation) versus angle of insonification for human myocardium. The insonification of representative epicardial, midmyocardial, and endocardial transmural levels are depicted. Each circumferential scan is initiated at the apical reference point (located at 0 degrees). The shift in the peaks of backscatter relative to the fixed reference point reflect a transmural shift of fiber orientation from epicardial to endocardial layers. layers. The average angular shift in the positions in the peaks and nadirs for consecutive transmural layers for human tissues was $9.2 \pm 0.7$ degrees $/ \mathrm{mm}$ for all tissue samples (Table I). Comparison of the fiber orientations with the position of the apical fiducial mark on the tissue plugs revealed that epicardial fibers coursed obliquely toward the apex, midmyocardial fibers maintained a circumferential orientation, and endocardial fibers coursed obliquely toward the base.

The average integrated backscatter for all angles of interrogation and for all transmural levels was $-53.4 \pm 0.4 \mathrm{~dB}$. Previously reported values for integrated backscatter of fresh canine myocardium measured without respect to fiber angle range from $\sim-50$ to $-60 \mathrm{~dB}(8-11)$. Concordance of measurements in fresh canine and fixed human tissues likely reflects the similar overall structural composition of human and canine myocardial tissue, whether fresh or fixed, and provides support for the use of fixed tissues for these measurements.

Control ultrasonic studies. The possible impact of artifact associated with the procedure for data acquisition necessitated consideration of the geometry of the interaction of the ultrasound beam with the small piece of heart tissue. The $-6-d B$ diameter of the broadband beam at the focal point was calculated as $2.03,1.22$, and $0.87 \mathrm{~mm}$ for $3-, 5-$, and $7-\mathrm{MHz}$ ultrasound, respectively, for the main lobe of a Bessel function for the transducer used. To determine experimentally whether the styrofoam holder might affect the measurement of integrated backscatter, circumferential scans were performed at selected levels below the edge of the holder with the holder in its usual position but with no tissue affixed. In the absence of tissue, ultrasonic interrogation revealed no significant backscatter above the noise level. No appreciable backscatter signal was observed within the gated rf segment even for scans centered as close as $1 \mathrm{~mm}$ from the edge of the styrofoam holder, which implies that sidelobe artifact was negligible at this range. With tissue in place, scans conducted in close proximity to either edge of the tissue (centered within $0.5 \mathrm{~mm}$ ) revealed obvious degradation of the signal with substantial increases in error bars around the mean values for the five spatially averaged sites. Therefore, all scans were centered at least $1 \mathrm{~mm}$ from the epicardial or endocardial edge of the tissue to maintain adequate clearance from the tissue edges over the useful frequency range.

An additional control experiment was performed to validate the scanning protocol by measurement of the angle dependence of scattering in a $3.0-\mathrm{mm}$ layer of pericardial fat in one cylindrical tissue sample. Fig. 5 shows that the amplitude of backscatter from every angle of insonification within the fat layer was essentially equivalent. In contrast, scans within myocardial tissue indicated the presence of substantial anisotropy of scattering, as described below. Thus, the scanning protocol appeared to provide a reliable method for differentiation of tissues expected to exhibit anisotropic scattering (myocardium) as compared with tissues expected to appear acoustically isotropic (fat).

To include data from the largest possible volume of tissue and to acquire data symmetrically about the point of rotation, we selected a gate length of $14 \mu \mathrm{s}$. The effect of gate length on the measurement of anisotropy was examined by varying the position and duration of the gated $\mathrm{rf}$ passed to the spectrum analyzer in one tissue sample at a single transmural level. Systematically deeper positioned 3- $\mu$ s gates were compared to a 14- $\mu \mathrm{s}$ gate. For 3- $\mu \mathrm{s}$ gates located toward the front, middle, and back of the specimen, anisotropy was equivalent, although the 
Table I. Transmural shift of fiber orientation calculated by ultrasonic and histologic methods for human myocardium

\begin{tabular}{|c|c|c|c|c|}
\hline \multirow[b]{2}{*}{ Sample } & \multicolumn{2}{|c|}{ Fiber angle shift } & \multirow{2}{*}{$\begin{array}{l}\text { Magnitude of } \\
\text { anisotropy }\end{array}$} & \multirow{2}{*}{$\begin{array}{c}\text { Average } \\
\text { backscatter }\end{array}$} \\
\hline & Ultrasound & Histology & & \\
\hline & \multicolumn{2}{|c|}{ degrees $/ \mathrm{mm}$} & \multicolumn{2}{|c|}{$d B$} \\
\hline H1 & $14.7 \pm 5.3(2)$ & $6.4 \pm 0.3(2)$ & $11.4 \pm 0.8(3)$ & $-53.3 \pm 0.7(3)$ \\
\hline $\mathbf{H} 2$ & $8.0 \pm 1.0(4)$ & $8.9 \pm 3.2(4)$ & $15.7 \pm 0.6(5)$ & $-50.7 \pm 0.5(5)$ \\
\hline H3 & $9.6 \pm 1.7(5)$ & $6.1 \pm 2.1(2)$ & $12.9 \pm 0.3(6)$ & $-55.0 \pm 0.7(6)$ \\
\hline H4 & $8.0 \pm 0.0(2)$ & $7.3 \pm 0.5(3)$ & $13.5 \pm 0.1(3)$ & $-51.4 \pm 0.3(3)$ \\
\hline H5 & $9.8 \pm 0.8(4)$ & $10.7 \pm 2.1$ & $15.3 \pm 0.7(5)$ & $-55.9 \pm 0.3(5)$ \\
\hline H6 & $6.7 \pm 1.3(2)$ & $7.8 \pm 2.0(2)$ & $10.7 \pm 0.7(3)$ & $-55.1 \pm 0.3(3)$ \\
\hline H7 & $8.0 \pm 1.3(3)$ & $6.8 \pm 0.5(3)$ & $20.5 \pm 1.1(4)$ & $-51.8 \pm 0.2(4)$ \\
\hline Totals & $9.2 \pm 0.7(22)$ & $7.9 \pm 0.8(19)$ & $14.5 \pm 0.6(29)$ & $-53.4 \pm 0.4(29)$ \\
\hline
\end{tabular}

The number of determinations of each index is shown in parentheses.

additional attenuation encountered for measurements at progressively more distant gated sites resulted in an expected reduction in the angle-averaged integrated backscatter. Thus, gate lengths as short as $3 \mu \mathrm{s}$ appear to permit a reasonable estimate of anisotropy for human tissues.

Comparison of ultrasound with histology. A total of 27 transmural sections were prepared for histologic analysis. One epicardial section was not used because a predominant fiber orientation could not be determined. Results of ultrasonic and histologic analyses of the fiber orientation for human myocardium are compared in Fig. 6. The peaks and nadirs of integrated backscatter were aligned closely with perpendicular and parallel fiber orientation, respectively, as judged by histologic analysis, which also revealed a progressive shift in myofiber direction from epicardium to endocardium of $7.9 \pm 0.8$ degrees/ $\mathrm{mm}$ of tissue. Ultrasonic and histologic analyses did not differ significantly with respect to the computed shift of myofiber direction from epicardium to endocardium. The concordance between ultrasonic and histologic methods for determination of fiber angle shift from endocardium to epicardium attests to the accuracy of ultrasonic measurements of myofiber orientation at selected transmural levels.

\section{Discussion}

Anisotropic ventricular myofiber architecture is a fundamental physiologic determinant of the mechanical and electrical func-

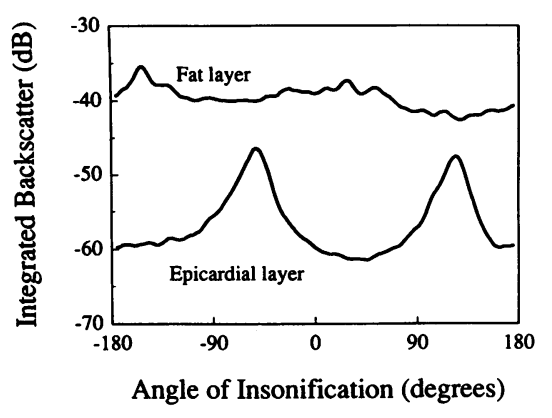

Figure 5. Apparent integrated backscatter versus angle of insonification for pericardial fat versus a representative example of epicardial tissue. No significant anisotropy of apparent integrated backscatter is observed for pericardial fat.

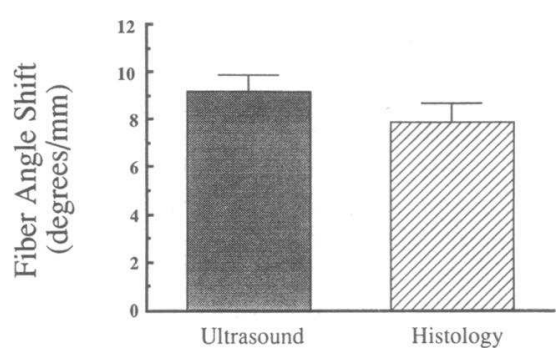

Method of Analysis

Figure 6. Comparison of results with histologic and ultrasonic methods for measuring fiber orientation. Both methods provide similar measures of the transmural shift of fiber orientation (in degrees per millimeter tissue) from epicardium to endocardium.

tion of the heart. The structural organization of materials such as heart tissue that are composed of highly aligned scattering elements may be characterized with ultrasound because the magnitude of ultrasonic scattering depends in part on the angle of insonification. Clinical evidence for ultrasonic anisotropy of myocardium has been reported recently by Aygen and Popp in two-dimensional echocardiographic images from normal patients as echo "drop-out" in certain views (22). Ultrasonic anisotropy also has been reported for other tissues such as skeletal muscle (27), kidney (28), and tendon (29). However, quantitative ultrasonic characterization of the three-dimensional intramural structure of human myocardial tissue has not been reported previously to our knowledge.

Our data indicate that the directional dependence of ultrasonic integrated backscatter at selected transmural levels reflects the orientation of myocardial fibers. Furthermore, the progressive shift in fiber orientation from epicardium to endocardium, as described by Streeter et al. $(1,2)$, can be defined by tissue characterization. The similarity of ultrasonic and histologic indexes of fiber orientation indicates that quantitative ultrasonic tissue characterization methods may be useful for delineating three-dimensional ventricular architecture at the level of cardiac myofibers and for detecting intramural structural detail not apparent by other imaging modalities.

The observation of ultrasonic anisotropy in cardiac tissue can be interpreted in light of well-defined physical models for the interaction of ultrasound with aligned scattering elements. For example, industrial composite materials that are constructed by embedding aligned graphite fibers in an epoxy medium exhibit substantial anisotropy of scattering and attenuation (30). Previously, we have constructed a tissue-mimicking phantom of oriented graphite fibers $(8-\mu \mathrm{m}$ diam) in gelatin whose structure is analogous to that of cardiac tissue at selected transmural levels with respect to the presence of tightly packed, highly oriented scatters. Both excised cardiac tissues and tissuemimicking phantoms exhibit maximal backscatter when insonified perpendicular to fibers and maximal attenuation and speed of sound when insonified parallel to fibers $(20,21,31-$ 33). We have extended these observations to beating hearts in open-chest dogs, which also manifest greater backscatter for insonification more perpendicular than parallel to midmyocardial fibers at all intervals during systole and diastole (21).

Mathematical analysis of the interactions of ultrasound with a collection of highly aligned scatterers has facilitated elucidation of the mechanisms of anisotropic scattering from 
myocardial tissue. Mottley and Miller (20) have modeled the scattering behavior of cardiac myofibers with the use of a timedomain Born approach for weakly scattering cylinders that was originally developed by Rose and Richardson (34). This formalism specifies the angle-dependent magnitude of scattering as a function of scatterer elasticity, density, geometry, and ultrasonic frequency. Elastic or geometric parameters that manifest a directional dependence represent primary determinants of the magnitude of scattering. Indeed, application of the timedomain Born approach to cardiac tissue indicates that greater backscatter is predicted for insonification perpendicular as compared with parallel to the predominant orientation of myofibers (20).

Because ultrasonic attenuation varies as a function of angle of insonification for canine myocardium, the observation of reduced backscatter for parallel insonification could be explained as a consequence of augmented attenuation. That is, less ultrasound would be available for scattering because of enhanced attenuation in the parallel direction. However, the magnitude of attenuation only increases approximately twofold for insonification parallel to fibers (32), which is not sufficient to account for the diminution of backscatter observed in the parallel direction (see Appendix I for further derivations). Hence, attenuation cannot represent the sole mechanism for the ultrasonic anisotropy of backscatter observed this study. We chose to report these data as "apparent" backscatter uncompensated for attenuation because this measurement is most relevant to future clinical applications of tissue characterization techniques.

Clinical implications for measurement of ultrasonic anisotropy. The overall aim of ultrasonic tissue characterization is to define normal and pathologic cardiac structure and function based on analysis of ultrasonic if signals scattered from tissue itself rather than from signals reflected from tissue boundaries $(7,8,35)$. Quantitative ultrasonic indexes of backscatter and attenuation have been used to elucidate structural and functional alterations associated with myocardial infarction, contusion, ischemic injury, and cardiomyopathy in experimental animals and patients $(7-19,35)$. Recently, our group has reported the development and clinical implementation of a two-dimensional ultrasonic backscatter imaging system that permits ultrasonic tissue characterization in real time at a patient's bedside $(16,17,19,25,26)$. Clinical tissue characterization with integrated backscatter can differentiate normal myocardium from cardiomyopathic, infarcted, and "stunned" myocardium in patients $(16,17,19)$. Clinical studies by Masuyama et al. at Stanford have used the backscatter imaging system developed at Washington University to characterize alterations in cardiac function associated with left ventricular hypertrophy, age, and cardiac transplant rejection (36-38). Vandenberg et al. also have used this backscatter imaging system in patients to detect regional differences in backscatter that appear to manifest a dependence on the specific view used for imaging (39).

We anticipate that clinical application of tissue characterization for measurement of ultrasonic anisotropy will provide important information about normal ventricular architecture and about pathologic processes that disrupt normal myofiber geometry and orientation. Fig. 7, $a$ and $b$, demonstrates that currently available ultrasonic tissue characterization methods can be used to measure ultrasonic anisotropy of myocardium from standard echocardiographic windows. The conventional two-dimensional echocardiographic image (Fig. 7 a) and a corresponding real-time integrated backscatter image (Fig. 7 b) were acquired in a normal volunteer from a parasternal shortaxis view at end-diastole with the use of a modified version of the clinical integrated backscatter imaging system previously described by our laboratory $(17,19,26)$. The quantitative backscatter image is displayed in gray scale and represents a linear dynamic range of $\sim 60 \mathrm{~dB}$ coded in 1-dB steps per gray level. The average magnitude of backscatter can be measured for any user-defined region of interest as small as $\sim 2 \mathrm{~mm}^{2}$ within the ventricular wall.

In the parasternal short-axis backscatter image (Fig. 7 b), the septum (SW) manifests greater integrated backscatter (brighter echoes) from right and left ventricular endocardial regions than from midmyocardial regions (darker). The values for backscatter from the two endocardial regions exceed those from the midmyocardial region by $\sim 5-15 \mathrm{~dB}$. One would predict less backscatter for insonification of midmyocardial septal fibers from a short-axis approach because sound waves propagate in a direction relatively parallel to the long axis of these circumferentially oriented myofibers in this view (see Discussion above). One would predict greater backscatter for insonification of endocardial fibers because sound waves propagate more perpendicular to the long axis of the obliquely oriented endocardial fibers.

The magnitude of integrated backscatter from the posterior wall (PW) appears more uniform from epicardium to endocardium (Fig. 7 b). The values for backscatter from either endocardial region in the posterior wall differ by $<3 \mathrm{~dB}$ from those of the midmyocardial region. One would predict more uniform backscatter for insonification of the posterior fibers because fibers from all parts of the wall are interrogated relatively perpendicular to their long axes. This is so because changes in orientation of fibers from epicardium to endocardium always occur within a plane that remains perpendicular to the direction of insonification in this view. Similar observations and predictions apply for the lateral wall and for apical twochamber, four-chamber, and subcostal views.

Potential clinical limitations. The major limitation of clinical ultrasonic tissue characterization is the quality of the primary data, which is determined not only by the patient but also by the operator. We estimate the percentage of transthoracic studies acquired from multiple views with our present equipment that are adequate for determination of backscatter to range between 50 and $90 \%$, depending on the clinical condition of the patient and the hospital setting. Because backscatter analysis can be performed on-line at the patient's bedside, it is easy to recognize immediately in which patients images suitable for analysis will be obtained.

For this study, we chose to measure backscatter from excised heart tissue to provide optimal baseline data necessary for eventual interpretation of clinical results. Whereas the measurement of backscatter in vitro allows determination of the absolute magnitude of scattering compensated for the effects of intervening attenuation, our current technique for clinical backscatter imaging permits accurate determination of relative magnitudes of scattering from selected myocardial regions. The potentially modest influence of attenuation on the measurement of anisotropy in this report is examined in Appendix I. Fortunately, the determination of the transmural shift of myofiber orientation only requires a measurement of the relative change in the magnitude of backscatter across the ventricular wall within a small myocardial region. The attenuation of 

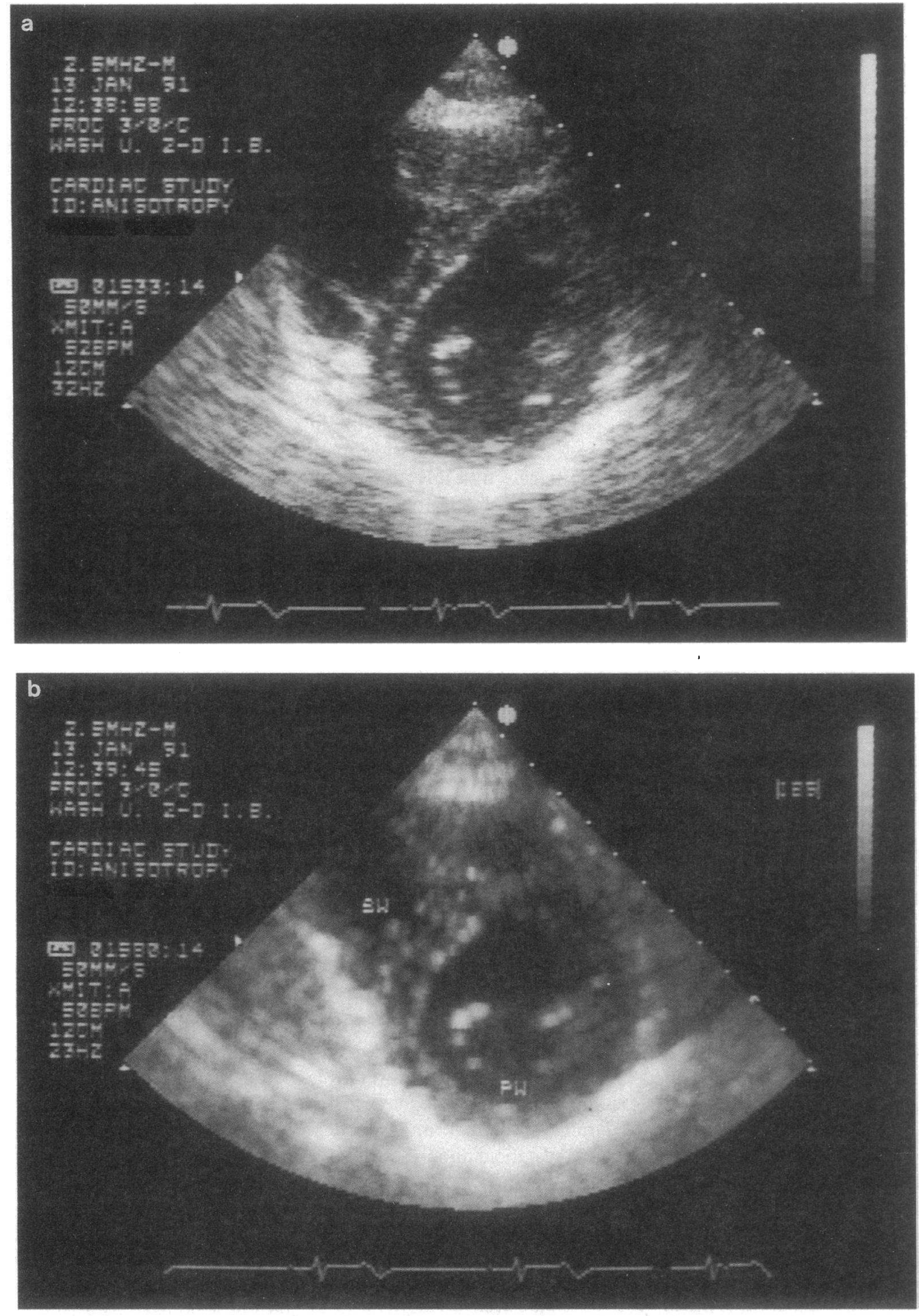

Figure 7. Conventional two-dimensional echocardiographic image (a) and a corresponding real-time quantitative integrated backscatter image $(b)$ of a normal volunteer subject from a parasternal short-axis view. Clinical ultrasonic tissue characterization methods can detect the dependence of backscatter on myofiber orientation (see text for description). The septum , $S W$ ) and posterior wall $(P W)$ are indicated in the backscatter image $(b)$.

ultrasound along the path to contiguous epicardial, midmyocardial, or endocardial zones is unlikely to differ sufficiently to vitiate the measurement of anisotropy.

The experimental procedure used for analysis of excised tissues in this study entailed circumferential scanning through $360^{\circ}$ at any transmural level to provide an exceptionally complete set of data for calculation of the transmural shift in fiber orientation. For clinical measurements of anisotropy, use of the conventional windows for transthoracic imaging will pro- vide a reduced but sufficient data set. Clinical echocardiographic imaging can be conducted from several orthogonal views that encompass both parallel and perpendicular directions of insonification for much of the left ventricle. Although the transmural fiber shift can be estimated from a single view within a local myocardial region of interest (Fig. 7 b), additional orthogonal views provide complementary data.

The ultimate use of tissue characterization for quantitative noninvasive analysis of cardiac histopathology, such as the 
structural alterations associated with myocardial infarction, will necessitate thorough delineation of physiologic and pathologic anisotropic properties that influence measurements of integrated backscatter. We anticipate that physiologic myocardial anisotropy can be mapped from standard transthoracic echocardiographic views to provide a complete clinical database for comparison with anisotropy under pathologic conditions. It is our hope that further refinements of this approach for studying three-dimensional myocardial architecture will permit noninvasive quantification of myofiber geometric and regional elastic characteristics.

\section{Appendix I}

In this section we estimate the potential influence of ultrasonic attenuation on the anisotropy of apparent integrated backscatter. The lumped attenuation parameter $|A(f)|^{2}$ specified in Methods can be expanded to account separately for the attenuation of ultrasound through tissue intervening between the surface of the specimen and the front of the gated volume, or $|I(f)|^{2}=\left(e^{-4 d a(f)}\right)$, and for the attenuation within the gated volume itself, or $|G(f)|^{2}=\left(1-e^{-4 \operatorname{sa}(f)}\right) /[4 g \alpha(f)]$, where $\alpha(f)$ is the frequency-dependent amplitude attenuation coefficient, $d$ is the length of tissue intervening between the front surface and the gate, and $g$ is the gate length. The total attenuation correction is $|A(f)|^{2}$ $=|I(f)|^{2}|G(f)|^{2}$. The apparent backscatter transfer function, $|B(f)|^{2}$, can be expressed in terms of decibels below the backscatter from a perfect reflector:

$10 \log |B(f)|^{2}=10 \log \left[|E(f)|^{2} /\left|E_{\text {red }}(f)\right|^{2}\right]$

$=10 \log \left[|I(f)|^{2}|G(f)|^{2}|S(f)|^{2}\right]$

.The intrinsic backscatter transfer function, $S(f)$, may be computed by correcting the apparent transfer function by the total attenuation. The frequency average of $10 \log |B(f)|^{2}$ represents apparent integrated backscatter, relative to scattering from a steel plate, and the frequency average of $10 \log |S(f)|^{2}$ represents intrinsic integrated backscatter.

Realistic choices for the slope of attenuation, $\beta(f)$, perpendicular and parallel to myocardial fibers are 0.55 and $1.2 \mathrm{~dB} / \mathrm{cm}$ per $\mathrm{MHz}$, respectively, based on previous studies by Mottley for canine myocardium (35). Here we make the common approximation: $\alpha=\beta f$. For a speed of sound in heart tissue of $\sim 1.55 \mathrm{~mm} / \mu \mathrm{s}, g=1.08 \mathrm{~cm}$ and $d$ $=0.16 \mathrm{~cm}$, the average attenuation, $\overline{|A(f)|^{2}}$, for the frequency band of interest (3-7 MHz) can be estimated for insonification either parallel or perpendicular to fibers as

$\overline{|A(f)|^{2}}=\frac{1}{N} \sum_{i=1}^{N} 10 \log \left[\left|I\left(f_{i}\right)\right|^{2}\left|G\left(f_{i}\right)\right|^{2}\right]$,

where $N$ is the number of estimates of attenuation from 3-7 MHz. The correction for average attenuation perpendicular to fibers is $3.9 \mathrm{~dB}$ and that parallel to fibers is $6.8 \mathrm{~dB}$. The excess average attenuation for insonification parallel as compared with perpendicular to fibers is approximately $(6.8-3.9 \mathrm{~dB})=3.9 \mathrm{~dB}$, which is significantly less than the magnitude of anisotropy ( $14.5 \mathrm{~dB})$ observed for integrated backscatter from human tissue. Thus, anisotropy of attenuation does not account for the major portion of the apparent anisotropy of integrated backscatter observed for myocardial tissue based on the values assumed for the slopes of attenuation for insonification parallel and perpendicular to fibers.

\section{Acknowledgments}

We greatly appreciate the assistance of Jeffrey Saffitz, M.D., Ph.D., in the acquisition of pathologic specimens, Andy Wong, M.D., and Michael Winters for their help with analysis of histologic data, Beth Engeszer for editorial assistance, and Ellen Visse for preparation of the manuscript.
Supported in part by grants HL42950, HL40302, HL17646, and a Clinician-Scientist Award from the American Heart Association (S. A. Wickline).

\section{References}

1. Streeter, D. D., R. N. Vaishnav, D. J. Patel, H. M. Spotnitz, J. R. Ross, Jr., and E. H. Sonnenblick. 1970. Stress distribution in the canine left ventricle during systole and diastole. Biophys. J. 10:345-363.

2. Streeter, D. D., Jr., and W. T. Hanna. 1973. Engineering mechanics for successive states in canine left ventricular myocardium. II. Fiber angle and sarcomere length. Circ. Res. 33:656-664.

3. Pierce, W. H. 1981. Body forces and pressures in elastic models of the myocardium. Biophys. J. 34:35-59. 288.

4. Chadwick, R. S. 1982. Mechanics of the left ventricle. Biophys. J. 39:279-

5. Chadwick, R. S., J. Ohayon, and M. Lewkowicz. 1989. Wall thickness and midwall-radius variations in ventricular mechanics. Proc. Natl. Acad. Sci. USA. 86:2996-2999.

6. Humphrey, J. D., and F. C. Yin. 1989. Constitutive relations and finite deformations of passive cardiac tissue: stress analysis in the left ventricle. Circ. Res. 65:805-817.

7. Miller, J. G., J. E. Perez, and B. E. Sobel. 1985. Ultrasonic characterization of myocardium. Prog. Cardiovasc. Dis. 28:85-110.

8. Perez, J. E., J. G. Miller, S. A. Wickline, M. R. Milunski, B. Barzilai, and B. E. Sobel. 1990. Myocardial tissue characterization. Prog. Cardiol. 3:83-96.

9. Wickline, S. A., L. J. Thomas III, J. G. Miller, B. E. Sobel, and J. E. Pérez. 1985. The dependence of myocardial ultrasonic backscatter on contractile performance. Circulation. 72:183-192.

10. Wickline, S. A., L. J. Thomas III, J. G. Miller, B. E. Sobel, and J. E. Pérez. 1985. A relationship between ultrasonic integrated backscatter and myocardial contractile function. J. Clin. Invest. 76:2151-2160.

11. Wickline, S. A., L. J. Thomas III, J. G. Miller, B. E. Sobel, and J. E. Perez. 1986. Sensitive detection of the effects of reperfusion on myocardium by ultrasonic tissue characterization with integrated backscatter. Circulation. 74:389400.

12. Chandraratna, P. A. N., R. Ulene, A. Nimalasuriya, C. L. Reid, D. Kawanishi, and S. H. Rahimtoola. 1985. Differentiation between acute and healing myocardial infarction by signal averaging and color encoding two-dimensional echocardiography. Am. J. Cardiol. 56:381-384.

13. Hoyt, R. H., S. M. Collins, D. J. Skorton, E. E. Ericksen, and D. Conyers. 1985. Assessment of fibrosis in infarcted human hearts by analysis of ultrasonic backscatter. Circulation. 71:740-744.

14. Wear, K. A., T. A. Shoup, and R. L. Popp. 1986. Ultrasonic characterization of canine myocardial contraction. IEEE (Inst. Electr. Electron. Eng.) Trans. UFFC-33:347-353.

15. Sagar, K. B., L. E. Pelc, T. L. Rhyne, S. Wann, and D. C. Waltier. 1988. Influence of heart rate, preload, afterload, and inotropic state on myocardial ultrasonic backscatter. Circulation. 77:478-483.

16. Vered, Z., B. Barzilai, G. A. Mohr, L. J. Thomas III, R. Genton, B. E. Sobel, T. A. Shoup, H. E. Melton, J. G. Miller, and J. E. Pérez. 1987. Quantitative ultrasonic tissue characterization with real-time integrated backscatter imaging in normal human subjects and in patients with dilated cardiomyopathy. Circulation. 76:1067-1073.

17. Vered, Z., G. A. Mohr, C. J. Gessler, S. A. Wickline, B. Barzilai, K. A Wear, T. A. Shoup, A. N. Weiss, B. E. Sobel, J. G. Miller, and J. E. Pérez. 1989. Ultrasound integrated backscatter tissue characterization of remote myocardial infarction in human subjects. J. Am. Coll. Cardiol. 13:84-91.

18. Milunski, M. R., G. A. Mohr, K. A. Wear, B. E. Sobel, J. G. Miller, and S. A. Wickline. 1989. Early identification with ultrasonic integrated backscatter of viable but stunned myocardium in dogs. J. Am. Coll. Cardiol. 14:462-471.

19. Milunski, M. R., G. A. Mohr, C. J. Gessler, Z. Vered, K. A. Wear, B. E. Sobel, J. G. Miller, J. E. Pérez, and S. A. Wickline. 1989. Ultrasonic tissue characterization with integrated backscatter. Acute myocardial ischemia, reperfusion, and stunned myocardium in patients. Circulation. 80:491-503.

20. Mottley, J. G., and J. G. Miller. 1988. Anisotropy of the ultrasonic backscatter of myocardial tissue. I. Theory and measurements in vitro. J. Acoust. Soc. Am. 83:755-761.

21. Madaras, E. I., J. E. Pérez, B. E. Sobel, J. G. Mottley, and J. G. Miller. 1988. Anisotropy of the ultrasonic backscatter of myocardial tissue. II. Measurements in vivo. J. Acoust. Soc. Am. 83:762-769.

22. Aygen, M., and R. L. Popp. 1987. Influence of the orientation of myocardial fibers on echocardiographic images. Am. J. Cardiol. 60:147-152.

23. Sigelmann, R. A., and J. M. Reid. 1973. Analysis and measurement of ultrasonic backscattering from an ensemble of scatterers excited by sine-wave bursts. J. Acoust. Soc. Am. 53:1351.

24. Miller, J. G., J. E. Perez, J. G. Mottley, E. I. Madaras, P. H. Johnston, E. D. Blodgett, L. J. Thomas III, and B. E. Sobel. 1983. Myocardial tissue charac- 
terization: an approach based on quantitative backscatter and attenuation. Proc IEEE Ultrasonics Symp. 83 CH 1947-1:782-793.

25. Thomas III, L. J., B. Barzilai, J. E. Pérez, B. E. Sobel, S. A. Wickline, and J. G. Miller. 1989. Quantitative real-time imaging of myocardium based on ultrasonic integrated backscatter. IEEE Trans. 36:466-470.

26. Wickline, S. A., and B. E. Sobel. 1989. Ultrasonic tissue characterization: prospects for clinical cardiology. J. Am. Coll. Cardiol. 14:1709-1711.

27. Wild, J. J., and J. M. Reid. 1953. The effects of biological tissues on 15-mc pulsed ultrasound. J. Acoust. Soc. Am. 25:270-280.

28. Rubin, J. M., P. L. Carson, and C. R. Meyer. 1988. Anisotropic ultrasonic backscatter from the renal cortex. Ultrasound Med. Biol. 14:507-511.

29. Fornage, B. 1987. The hypoechoic normal tendon-a pitfall. J. Ultrasound Med. 6:19-22.

Blodgett, E. D., L. J. Thomas III, and J. G. Miller. 1986. Effects of porosity on polar backscatter from fiber reinforced composites. Rev. Prog. Quant. Nondestructive Eval. 5B:1267-1274.

31. Mol, C. R., and P. A. Breddels. 1982. Ultrasound velocity in muscle. $J$. Acoust. Soc. Am. 71:455-461.

32. Mottley, J. G., and J. G. Miller. 1990. Anisotropy of the ultrasonic attenuation in soft tissues: measurements in vitro. J. Acoust. Soc. Am. 88:1203-1210.

33. Mottley, J. G. 1985. Physical principles of the ultrasonic attenuation and backscatter of soft tissues: dependence on the angle of propagation and the physiologic state. Ph.D. dissertation. Washington University, St. Louis, MO.
34. Rose, J. H., and J. M. Richardson. 1982. Time domain Born approximation. J. Nondestructive Eval. 3:45-53.

35. Skorton, D. J., J. G. Miller, S. A. Wickline, B. Barzilai, S. M. Collins, and J. E. Perez. 1991. Ultrasonic characterization of cardiovascular tissue. Chapter 26 In Cardiac Imaging: A Companion to Braunwald's Heart Disease. M. L. Marcus, H. R. Schelbert, D. J. Skorton, and G. Wolf, editors. W. B. Saunders Co., Philadelphia, PA. 538-556.

-36. Masuyama, T., U. Nellessen, I. Schnittger, T. L. Tye, W. L. Haskell, and R. L. Popp. 1989. Ultrasonic tissue characterization with a real-time integrated backscatter imagining system in normal and aging human hearts. J. Am. Coll. Cardiol. 14:1702-1708.

37. Masuyama, T., F. G. St. Goar, T. L. Tye, G. Oppenheim, I. Schnittger, and R. L. Popp. 1989. Ultrasonic tissue characterization of human hypertrophied hearts in vivo with cardiac cycle-dependent variation in integrated backscatter. Circulation. 80:925-934.

38. Masuyama, T., H. A. Valentine, R. Gibbons, I. Schnittger, and R. L. Popp. 1990. Serial measurements of integrated backscatter in human cardiac allografts for the recognition of acute rejection. Circulation. 81:829-839.

39. Vandenberg, B. F., L. Rath, T. A. Shoup, R. E. Kerber, S. M. Collins, and D. J. Skorton. 1989. Cyclic variation of ultrasound backscatter in normal myocardium is view dependent: clinical studies with a real-time backscatter imaging system. J. Am. Soc. Echocardiogr. 2:308-314. 